\title{
Study of Clinico-Histopathological Correlation of Seborrheic Keratosis
}

\author{
Dharti Durlabhjibhai Jakasania* and Shaila N. Shah
}

Pathology Department, Government Medical college Bhavnagar, Gujarat, India 364001

\begin{abstract}
Background: Seborrheic keratosis (SK) is one of the mostcommon epidermal tumors of the skin. However, only a few large-scale clinichistopathological investigations have been conducted on SK or on the possible correlation be-tween histopathological SK subtype and location. The aim of this study was to analyze the clinical and histopathological features of a relatively large number of cases of diagnosed SK.

Methods: Seventy five (75) pathology slides of skin tissue from patients with clinically diagnosed SK and 67 cases of biopsy-proven SK were analyzed. The biopsy-proven cases of SK were assessed for histopathological subclassification. The demographic, clinical, and histopathological data of the patients were collected for analysis of associated factors.

Result: Seborrheic keratosis commonly present in old age most common age group is 60-70 yr. It affects male and female equally (M:F-1.12:1).Most common etiology in our study is sun exposure. In our study upper extremities are most commonly involved. Among histologically proven 67 cases most common histological type in our study is clinical seborrheic keratosis(CSK) (68.65\%) followed by stucco keratosis $(23.88 \%)$. Among common seborrheic keratosis hyperkeratotic type (31.34\%) is most common followed by melanoacanthoma $(19.40 \%)$.Histopathologically proven cases of seborrheic keratosis shows significant presence histopathological features like hyperkeratosis $(69.33 \%)$, acanthosis $(66.66 \%)$ and papillomatosis $(60 \%)$. Hyperpigmentation is also present in significant number of cases $(34.66 \%)$.

Conclusion: SK appear to be more common in old-age, with equal sex distribution, predominantly affecting the upper extremities, head, and neck. Sun exposure was the most common etiology.

The most frequent SK histopathological sub-type found in this study was the clinical seborrheic keratosis followed by stucco keratosis. In clinical seborrheic keratosis most common variant was hyperkeratotic type followed by melanoacanthoma. Most of the characteristics of SK are in accordance with the literature published.
\end{abstract}

Keywords: Seborrheic Keratoses, Dermatosis Papulosanigra, Histology.

\section{Introduction}

Seborrheic keratosis (SK) is one of the most common benign epidermal tumors that affects both sexes equally, and usually arise in individuals older than 50 years ${ }^{(1-4)}$.

It presents as sharply demarcated, slightly raised brownish patches or plaques ${ }^{(2,5)}$. They usually start off with a light tan and then may darken to dark brown or nearly black. The consistent feature of seborrheic keratosis is their waxy, pasted-on or stuck-on look. The look is often compared to brown candle wax dropped onto the skin. The condition is very common in white races, usually occurs in middle aged individuals and can arise as early as adolescence ${ }^{(3)}$.

The lesions of SK can occur at any site over the body, but are most frequent on the face and upper trunk (sun-exposed surfaces of the skin). The lesions are usually asymptomatic, but may be itchy ${ }^{(2)}$. The clinical presentation can be quite variable and includes clinical variants, such as stucco keratosis and dermatosis papulosa nigra. Although there are many clinical variants of the lesions, these lesions usually begin as well-circumscribed, dull, flat, tan, or brown patches. As they grow, they become more papular, taking on a waxy verrucous or "stuck-on" appearance ${ }^{(1)}$.

Despite the diverse clinical presentation of SK, diagnosis is most often clinically straightforward. However, the tumor may simulate other lesions, such as common warts, lentigines, melanocytic nevi, actinic keratosis, and Bowen disease, or occasionally more aggressive entities, such as basal cell and squamous cell carcinomas, or even cutaneous melanomas ${ }^{(6-8)}$.

It can be divided into six major histopathological variants: acanthotic, hyperkeratotic, adenoid, irritated, clonal, and melanoacanthoma.

Histopathologically, all of the subtypes have three features in common:

1 Hyperkeratosis, 
2 Acanthosis,

3 Papillomatosis.

On low- to moderate-power magnification, the base of the lesion lies roughly on an imaginary axis drawn between two dermoepidermal junctions at both ends of the surrounding normal tissue ${ }^{(2)}$.Even though SK is presently one of the most common cosmetic skin complaints, there is a lack of data relating to its clinical, dermoscopic and histopathologic correlation. This study may provide useful information regarding the etiology and pathology of SK.

\section{Materials and Methods}

The study was conducted in the Dermatology Out-patients Department, Government Medical College Bhavnagar, from April 2017 to August 2018. Institute ethical clearance was obtained before the study. Patients with clinical diagnosis of seborrheic keratosis, who were above 18 years and were willing to participate in the study, were included. A written informed consent was taken from all patients. Patients with SK were evaluated with respect to age, sex, age of onset, site of lesions, number of lesions, morphology, and symptoms associated with the lesions. An incisional or punch biopsy was taken from the lesions and assessed for histopathology. Tissue samples from 75 cases of clinically diagnosed SK (including 8 cases initially diagnosed clinically as SKs, but subsequently proven not to be on histological examination).

The medical records were investigated retrospectively for any difference in age, sex, site of occurrence, and analysis of the clinicopathological mismatch cases (i.e., cases in which there was mismatch between the clinical and pathological diagnoses).

Punch biopsy of 0.3 to $0.4 \mathrm{~cm}$ size was taken from the lesion site.

\section{Result}

In our study most common age group of presentation of Seborrheic keratosis is 60 to 70 year. In our study as mentioned earlier hyperkeratosis is most common feature all over as well as in CSK followed by acnthosis and papillomatosis. In our study in CSK horn cyst is least common feature. (Table-2)

Table 1: Age distribution of 67 biopsy-proven as seborrheic keratosis patients.

\begin{tabular}{|c|c|c|c|}
\hline Age group(year) & Male & Female & Total \\
\hline$<20$ & 00 & 00 & 00 \\
\hline $21-30$ & 00 & 00 & 00 \\
\hline $31-40$ & 00 & 05 & 05 \\
\hline $41-50$ & 05 & 07 & 12 \\
\hline $51-60$ & 09 & 09 & 18 \\
\hline $61-70$ & 12 & 09 & 21 \\
\hline $71-80$ & 07 & 03 & 10 \\
\hline$>80$ & 01 & 00 & 01 \\
\hline Total & $\mathbf{3 4}$ & $\mathbf{3 3}$ & $\mathbf{6 7}$ \\
\hline
\end{tabular}

Table 2: Comparison of different histological variants.

\begin{tabular}{|c|c|c|c|}
\hline & \multicolumn{3}{|c|}{ Our study } \\
\hline Acanthosis & CSK(\%) & Stucco(\%) & DPN(\%) \\
\hline Hyperkeratosis & 71.73 & 75 & 80 \\
\hline Papillomatosis & 82.6 & 18.75 & 100 \\
\hline Hyperpigmentation & 60.86 & 81.25 & 20 \\
\hline Horncyst or Pseudohorn cyst & 45.65 & 6.25 & 60 \\
\hline
\end{tabular}

Table 3: Comparison of histological features with other similar study.

\begin{tabular}{|c|c|c|c|}
\hline & Rajesh et al[9] \% & Geethu et al[10] \% & Our study\% \\
\hline Acanthosis & 56 & 56.50 & 66.66 \\
\hline Hyperkeratosis & 54 & 56.50 & 69.33 \\
\hline Papillomatosis & 68 & 78.30 & 60 \\
\hline Hyperpigmentation & 78 & 73.90 & 34.66 \\
\hline Horncyst or Pseudohorn cyst & 44 & 65.20 & 33.33 \\
\hline
\end{tabular}


Table 4: Comparison of different histological variants with other studies.

\begin{tabular}{|c|c|c|c|c|c|c|}
\hline & \multicolumn{3}{|c|}{ Geethu et al[10] } & \multicolumn{3}{c|}{ Our study } \\
\hline & $\begin{array}{c}\text { CSK } \\
(\%)\end{array}$ & $\begin{array}{c}\text { Stucco } \\
(\%)\end{array}$ & $\begin{array}{c}\text { DPN } \\
(\%)\end{array}$ & $\begin{array}{c}\text { CSK } \\
(\%)\end{array}$ & $\begin{array}{c}\text { Stucco } \\
(\%)\end{array}$ & $\begin{array}{c}\text { DPN } \\
(\%)\end{array}$ \\
\hline Acanthosis & 56.5 & 44.4 & 53.8 & 71.73 & 75 & 80 \\
\hline Hyperkeratosis & 56.5 & 44.4 & 53.8 & 82.6 & 18.75 & 100 \\
\hline Papillomatosis & 78.3 & 22.2 & 84.6 & 60.86 & 81.25 & 20 \\
\hline Hyperpigmentation & 73.9 & 100 & 84.6 & 45.65 & 6.25 & 60 \\
\hline Horncyst or Pseudohorn cyst & 65.2 & 55.6 & 7.7 & 47.82 & 00 & 40 \\
\hline
\end{tabular}

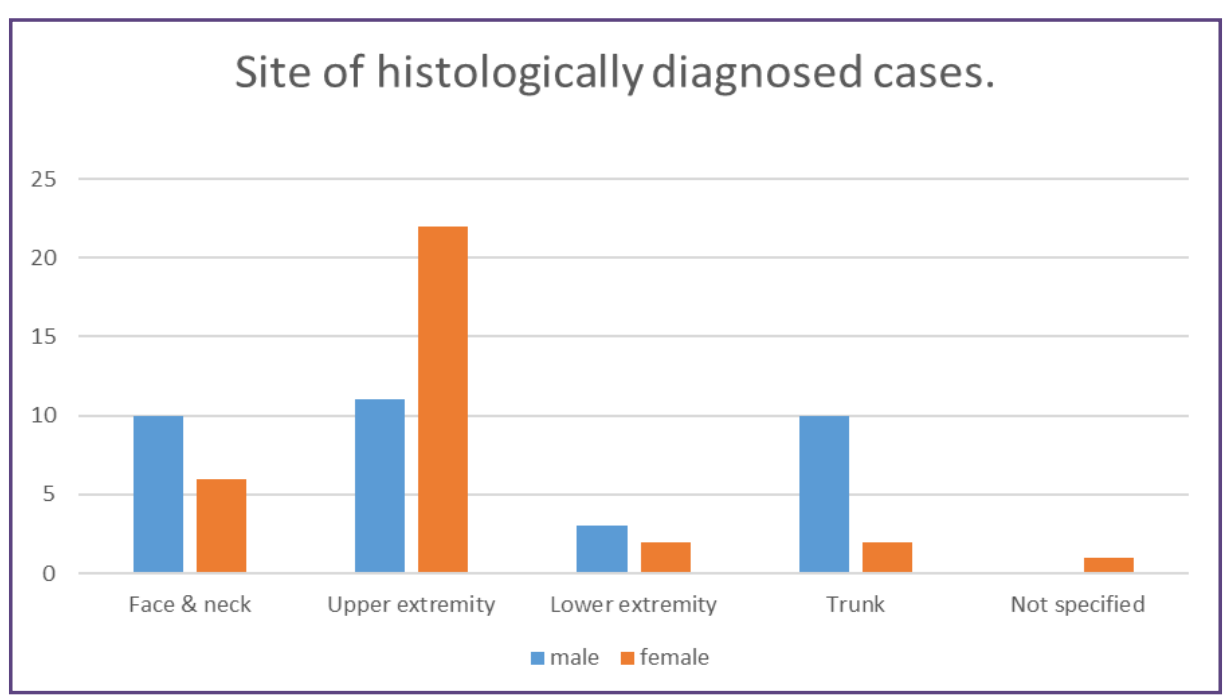

Fig. 1: Site of histologically proven cases.

According to site upper extremities are most commonly involved in our study. In females upper exytrimities are commonly involved while in male trunk is more commonly involved than any other site.(fig-1)

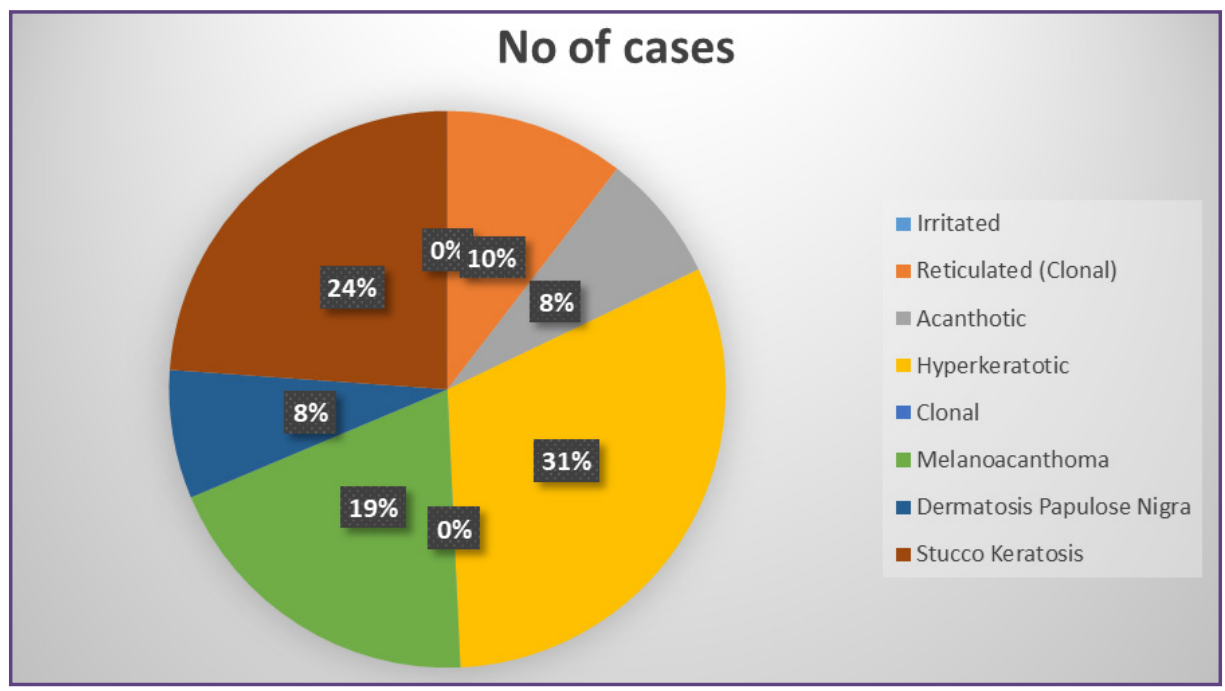

Fig-2. Distribution of histopathological subtypes in the 75 biopsy-proven cases of seborrheic keratosis.

In our study most common histological type is hyperkeratotic type followed by melanoacanthoma and reticulated(clonal) variant. Stucco keratosis cases are also in significant number.(fig-2) 


\section{Discussion}

This prospective study analyzed clinical and histopathological features of 75 skin biopsy cases clinically diagnosed as SKs and subclassified 67 skin biopsy cases confirmed as SKs according to their histopathological characteristics.

Sex ratio The findings of this study show that clinically diagnosed and biopsy-proven SKs affect men and women with almost equal frequencies, in agreement with the findings of other studies. $[3,12,15]$ Gender distribution of SK is balanced. ${ }^{[11]} \mathrm{A}$ male preponderance of $50.66 \%$ only by $0.66 \%$ seen in our study which is not at all significant. which is also supported by rajesh at al study. ${ }^{[3,9]}$

Sex distribution is almost equal in our study and M:F ratio is $1.12: 1$.

Age The most affected decade of age is 60, also as in other studies ${ }^{[1,12]}$, was the $60 \mathrm{~s}$, with both clinically diagnosed SKs and biopsy-proven SKs. When comparing affected ages among the studies. ${ }^{[1,12,13]}$ it appears that the mean affected age has increased over time- 52.2 years in the study of Ahn et al. (1980s) $)^{[13]}$ vs. 60.12 years in the present study, which is supported by Nam Kyung Roh study(60.9). ${ }^{[8]}$

Etiology Although the etiology of SK is currently unclear, it has been suggested that exposure to sunlight leads to the development of SKs. ${ }^{[3,4]}$ Thus, it may be that this apparent increase in SK-affected age over the past three decades is attributable to a reduction in the exposure to ultraviolet (UV) light due to the average amount of time spent indoors having also increased during this period. Many different factors from time to time have been implicated in the etiology of SK, but none is well understood. Genetics and Sun exposure have been implicated as the major causes. ${ }^{[14,16]}$

Majority of our patients had a positive history of Sun exposure was seen to be a major factor in our study as only $28 \%$ of our patients had lesions in covered areas such as chest, abdomen, back, thighs, and legs and remaining $72 \%$ have lesions in sun exposed areas like upper extremity, face and neck. Though its only according to taken biopsy data. However, lesion presents in almost $90 \%$ of cases on sun exposed areas. This was in favor to a study conducted on Korean males by Kwon et al. on 303 volunteers aged 40-70 years, which showed cumulative Sunlight exposure to be a contributing factor. ${ }^{[4]}$

Histology Subtype To the best of our knowledge, no previous studies have analyzed the histopathological subtypes of SK according to disease location. In our study, we analyzed histopathological subtype according to location, and the hyperkeratotic type was found to occur more commonly in sun-exposed sites (85.7\%). Thus, it appears that UV light plays a definite role in the development of at least the hyperkeratotic type of SK. Melanoacanthoma (69.3\%) also most commonly present over sun exposed area. Stucco keratosis's common presentation over sun exposed area especially over distal portion of extremity like hand, lowe leg etc it cover almost $86.66 \%$ of total case of stucco keratosis.

Clinical And Histopathological Correlation. The association between frequency of clinicopathological mismatch among clinically diagnosed SK patients and SK lesion location was also evaluated in the present study.

Each type of SK demonstrates varying degrees of acanthosis, pigmentation, horn cysts formation, hyperkeratosis, and papillomatosis, when viewed histologically. ${ }^{[2,17]}$

Our study also showed these to be the most common findings on histopathology with Hyperkeratosis (69.33\%) being the most common followed by acanthosis $(66.66 \%)$, papillomatosis (60\%), pigmentation (34.66\%), horn cyst $(33.33 \%)$, orthokeratosis $(32 \%)$, and parakeratosis $(17.33 \%)$ which is differ from Geethu Francis Alapatt et al study in which pigmentation (78\%) being the most common followed by papillomatosis $(68 \%)$, acanthosis $(56 \%)$, hyperkeratosis $(54 \%)$, and horn cyst formation $(44 \%){ }^{[10]}$

Our study has reported the frequency of occurrence of various clinical types of SK, CSK being the most common $(68 \%)$, followed by flat and pedunculated SK (12\%), and stucco keratoses $(6.66 \%)$. least common variant in our study is DPN(1.33\%). ${ }^{[9]}$ Geethu Francis Alapatt et al study also showed the highest incidence of CSK (46\%), followed by DPN (26\%). We had a higher incidence of stucco keratoses (18\%) compared to pedunculated variety $(10 \%){ }^{[10]}$

A study conducted in South India by Rajesh et al. has reported the frequency of occurrence of various clinical types of SK, CSK being the most common (60\%), followed by DPN (46.6\%), pedunculated SK (21.2\%), and stucco keratoses $(2 \%) .{ }^{[9]}$ Geethu Francis Alapatt et al also showed the highest incidence of CSK (46\%), followed by DPN $(26 \%)$.Study showed higher incidence of stucco keratoses $(18 \%)$ compared to pedunculated variety $(10 \%) .^{[10]}$

In our study hyperkeratosis is most common histological feature followed by acanthosis and papillomatosis, which is compared with other studies.(table-3)

The histopathological features seen in CSK in our study were hyperkeratosis (82.6\%), acanthosis(71.73\%), 
papillomatosis $(60.86 \%)$, pigmentation $(45.65 \%)$ and horn cysts $(47.82 \%)$. The presence of horn cysts was statistically significant $(\mathrm{P}=0.005)$. The histopathological features seen in stucco keratoses were papillomatosis $(81.25 \%)$, acanthosis and orthokeratosis (75\%), hyperkeratosis and parakeratosis (18.75\%) and pigmentation (6.5\%). The histopathological features seen in DPN were hyperkeratosis in $100 \%$, acanthosis $(80 \%)$ pigmentation $(60 \%)$, orthokeratosis and horn cyst (40\%) and papillomatosis $(20 \%)$.

The histopathological features seen in CSK in Geethu Francis Alapatt et al study were papillomatosis (78.30\%), pigmentation $(73.90 \%)$, horn cysts $(65.20 \%)$, acanthosis and hyperkeratosis $(56.50 \%)$. The histopathological features seen in DPN were papillomatosis, pigmentation $(84.6 \%)$, acanthosis and hyperkeratosis $(53.8 \%)$. Horn cysts were seen only in $7.7 \%$ of the cases. The histopathological features seen in stucco keratoses were pigmentation, which was present in $100 \%$ of the cases followed by the presence of horn cysts $(55.6 \%)$, acanthosis and hyperkeratosis (44.4\%), and papillomatosis in $22.2 \%$ of the cases. ${ }^{[10]}$

In our study as mentioned earlier hyperkeratosis is most common feature all over as well as in CSK followed by acnthosis and papillomatosis, which is different from other compared study in which papillomatosis is more common followed by pigmentation and horn cyst. In our study in CSK horn cyst is least common feature. As there is less number of cases of stucco(16) and DPN (5) comparision may not be validated.(Table-4)

\section{Clinical variants}

Common Seborrheic Keratosis: Common SK appear only on hair-bearing surfaces, predominantly on the hand, head, trunk, and neck. In our study, 76\% (51) of cases had CSK, the commonest of all variants that we had observed. The existing literature mentions that CSK are usually seen after the third decade, whereas the youngest patient with CSK in our study was a 33-yearold female, although only $8.6 \%$ of total cases $(n=13)$ with CSK were in the age group less than 40 years. Our study has reported increased frequency of CSK in males, with a M:F ratio of 1.12:1. which is supported by Geethu Francis Alapatt et al study in which ratio is $1.23: 1 .^{[9]}$

Pedunculated Seborrheic Keratosis: We observed clinically, another group of SK which are brown to black, pedunculated papules, of size $1-5 \mathrm{~mm}$, most commonly in areas of friction, commonly seen on face, neck and axillae, and categorized them as PSK. The $40 \%$ of cases with PSK were in fifth decade of life(50yr).

Flat Seborrheic Keratosis: Flat SK are benign lesions that typically appear as oval tan-brown patches or macules on sun-exposed areas of the skin, especially on the face, backs of the hands and wrists, extensor surfaces of the forearms, and chest. Flat SK are asymptomatic and increase with the age.[8] In our study,flat Sk patients present mostly in 60s or $70 \mathrm{~s}$ with almost equal sex distribution.

Stucco keratosis: White or gray-white verrucous papules are a few millimeters in size, often in large numbers on sun-exposed areas (extensor surface of forearms and lower legs, dorsal aspects of hands and feet). There were only five cases (two males and three females) of stucco keratoses in our study. These lesions were observed on hands and abdomen as mentioned in the literature.

\section{Conclusion}

Seborrheic Keratosis is a benign epidermal disease of skin. Seborrheic keratosis commonly present in old age most common age group is 60-70 yr. It affects male and female equally (M:F-1.12:1).

Most common etiology in our study is sun exposure because most lesions are present on sun exposed areas of body. In our study upper extremities are most commonly involved.

Among 75 clinically diagnosed seborrheic keratosis cases 67 histopathologically proven as seborrheic keratosis. $(89.33 \%)$

Among them histologically proven 67 cases most common histological type in my study is clinical seborrheic keratosis (68.65\%) followed by stucco keratosis $(23.88 \%)$. Among common seborrheic keratosis hyperkeratotic type $(31.34 \%)$ is most common followed by melanoacanthoma $(19.40 \%)$.

Histopathologically proven cases of seborrheic keratosis shows significant presence of its common histopathological features like hyperkeratosis $(69.33 \%)$, acanthosis $(66.66 \%)$ and papillomatosis $(60 \%)$. Hyperpigmentation is also present in significant number of cases $(34.66 \%)$.

\section{References}

1. Lee GS, Ahn KJ, Kim JM, Lee ES. A histopathologic study of the seborrheic keratosis. Korean Journal of Dermatology. 1992 Feb 1;30(1):76-80.

2. Pending Elder DE. Lever's histopathology of the skin. Lippincott Williams \& Wilkins; 2014 Sep 9.

3. Yeatman JM, Kilkenny M, Marks R. The prevalence of seborrhoeic keratoses in an Australian population: does exposure to sunlight play a part in their frequency? British Journal of Dermatology. 1997 Sep;137(3):411-4.

4. Kwon OS, Hwang EJ, Bae JH, Park HE, Lee JC, Youn JI, Chung JH. Seborrheic keratosis in the Korean males: causative role of sunlight. Photodermatology, photoimmunology \& photomedicine. 2003 Apr;19(2):73-80. 
5. Kim HY, Kim HS, Cho EB, Park EJ, Kim KH, Kim KJ. A clinicohistopathological study on the lesion resembling seborrheic keratoses of the face. Korean Journal of Dermatology. 2013 Jul 1;51(7):494-500.

6. Jeong YI, Lee WJ, Bak H, Oh SH, Jung HJ, Chang SE, Choi JH. Detection of human papilloma virus DNA in seborrheic keratosis of Korean skin. Annals of Dermatology. 2007 Sep 1;19(3):99-105.

7. Marks R, Jolley D, McCormack C, Dorevitch AP. Who removes pigmented skin lesions?: A study of the ratio of melanoma to other benign pigmented tumors removed by different categories of physicians in Australia in 1989 and 1994. Journal of the American Academy of Dermatology. 1997 May 1;36(5):721-6.

8. Roh NK, Hahn HJ, Lee YW, Choe YB, Ahn KJ. Clinical and histopathological investigation of seborrheic keratosis. Annals of dermatology. 2016 Apr 1;28(2):152-8.

9. Rajesh G, Thappa DM, Jaisankar TJ, Chandrashekar L. Spectrum of seborrheic keratoses in south Indians: A clinical and dermoscopic study. Indian Journal of Dermatology, Venereology, and Leprology. 2011 Jul 1;77(4):483.

10. Alapatt GF, Sukumar D, Bhat MR. A clinicopathological and dermoscopic correlation of seborrheic keratosis. Indian journal of dermatology. $2016 \mathrm{Nov} ; 61(6): 622$.
11. Zhang RZ, Zhu WY. Seborrheic keratoses in five elderly patients: An appearance of raindrops and streams. Indian journal of dermatology. $2011 \mathrm{Jul} ; 56(4): 432$.

12. Park S, Park H, Cho K. Clinical and histopathologic study of seborrheic keratosis. Korean Journal of Dermatology. 2011 Jan 1;49(1):12-9.

13. Roh NK, Hahn HJ, Lee YW, Choe YB, Ahn KJ. Clinical and histopathological investigation of seborrheic keratosis. Annals of dermatology. 2016 Apr 1;28(2):152-8.

14. Thomas VD. Benign epithelial tumors, hamartomas, and hyperplasias. Fitzpatrick's dermatology in general medicine. 2008.

15. Elgart GW. Seborrheic keratoses, solar lentigines, and lichenoid keratoses: dermatoscopic features and correlation to histology and clinical signs. Dermatologic clinics. 2001 Apr 1;19(2):347-57.

16. Moskaluk CA, Merino MJ, Danforth DN, Medeiros LJ. Lowgrade angiosarcoma of the skin of the breast: a complication of lumpectomy and radiation therapy for breast carcinoma. Human pathology. 1992 Jun 1;23(6):710-4.

17. Engel A, Johnson ML, Haynes SG. Health effects of sunlight exposure in the United States: results from the first National Health and Nutrition Examination Survey, 1971-1974. Archives of dermatology. 1988 Jan 1;124(1):72-9.

*Corresponding author:

Dr. Dharti Durlabhjibhai Jakasania, Resident Pathology 104, Pg-3 Hostel, Sir T. Hospital campus, Jail road, Bhavnagar, Gujarat, India. 364001.

Phone: +91 9429587720

Email: dharti.jakasania@gmail.com

Financial or other Competing Interests: None. 